\title{
Classification of Human Emotions using EEG Signals
}

\author{
Pravin Kshirsagar \\ Research Scholar, \\ Department of Electronics \\ Engineering, \\ Rajiv Gandhi College of \\ Engineering and Technology, \\ Chandrapur (M.S.) India
}

\author{
Sudhir Akojwar \\ Senior Member IEEE, \\ Professor, Department of \\ Electronics Engineering, \\ Rajiv Gandhi College of \\ Engineering and Technology, \\ Chandrapur (M.S.) India
}

\begin{abstract}
In this paper we proposed new features based on wavelet transform for classification of human emotions (disgust, happy, surprise, fear and neutral). from electroencephalogram (EEG) signals.EEG signals are collected using 64 electrodes from twenty subjects and are placed over the entire scalp using International 10-10 system or international 10-20 system. The EEG signals are preprocessed using filtering methods to remove the noise. Feature extraction of the principle signal is done by using methods such as wavelet transform. The feature extracted signals are then classified using Neural Network (NN) and the neural system is trained and we get trained classifier according to the classification of the signals and the results are obtained. To test the signal the feature extracted signals are given directly to the trained classifier and results are obtained.
\end{abstract}

\section{Keywords}

Electroencephalogram (EEG); Neural Network (NN); Wavelet transform

\section{INTRODUCTION}

The inner state of a person "Emotions" play a important or vital role in analyzing the state of mind [1].Emotion is one of the most essential features of humans. Without the capability of emotions processing, computers and robots are not communicates with human in natural way [2].Emotion is often interred twined with mood, temperament, personality, disposition, and motivation. Emotions are complex they are a state of feeling that results in physical and psychological changes that influence our behavior.

In emotion assessment using EEG signals, the time duration of EEG signals under given, number of channels, emotional stimuli, frequency bands, nature of statistical feature extraction methods and features plays an significant

Role. The traditional tools for the investigation of human

Emotional status are based on the statistical analysis and
Recording of physiological signals from the both central and autonomic nervous systems (CNS and ANS). In this, we are considered the signals like valance, arousal, dominance and liking. The emotional experiences can be subdivided into 2 dimensions called as valence (how negative or positive the experience feels) and arousal (how enervated or energized the experience feels). These 2 dimensions are depicted on a 2-D coordinate map [3]. This 2-dimensional map was theorized for capturing one essential component of emotion which is known as core affect.

Emotions can also been described as a biologically given and a result for evolution because they provided good solutions to ancient and recurring problems that faced our ancestors.

Feelings are best understood as a subjective representation of emotions, private to the individual experiences them. Moods have diffuse affective states that generally last for much longer durations than emotions and are also generally less intense than that of the emotions. Affect is an encompassing term, used to describe the topics of emotion, moods and feelings, together, even though it is commonly used interchangeably with emotion. In addition, relationships exist between emotions, like having positive or negative influences, with direct opposites existing .In addition, relationships exist between emotions, such as having positive or negative influences, with direct opposites existing.

Parts of the brain activated for different Emotion sets

Happy - The left side of the frontal lobe - the left prefrontal cortex.

Sad - The right side of the frontal lobe -the right prefrontal cortex.

Laughter and humor - Temporal lobe and hypothalamus Anger - Limbic center of the brain The small structure within the limbic system is termed as the amygdale. Fast eye moment sleep - It is the cerebral cortex. 


\section{FLOW CHART OF PROPOSED}

ALGORITHM

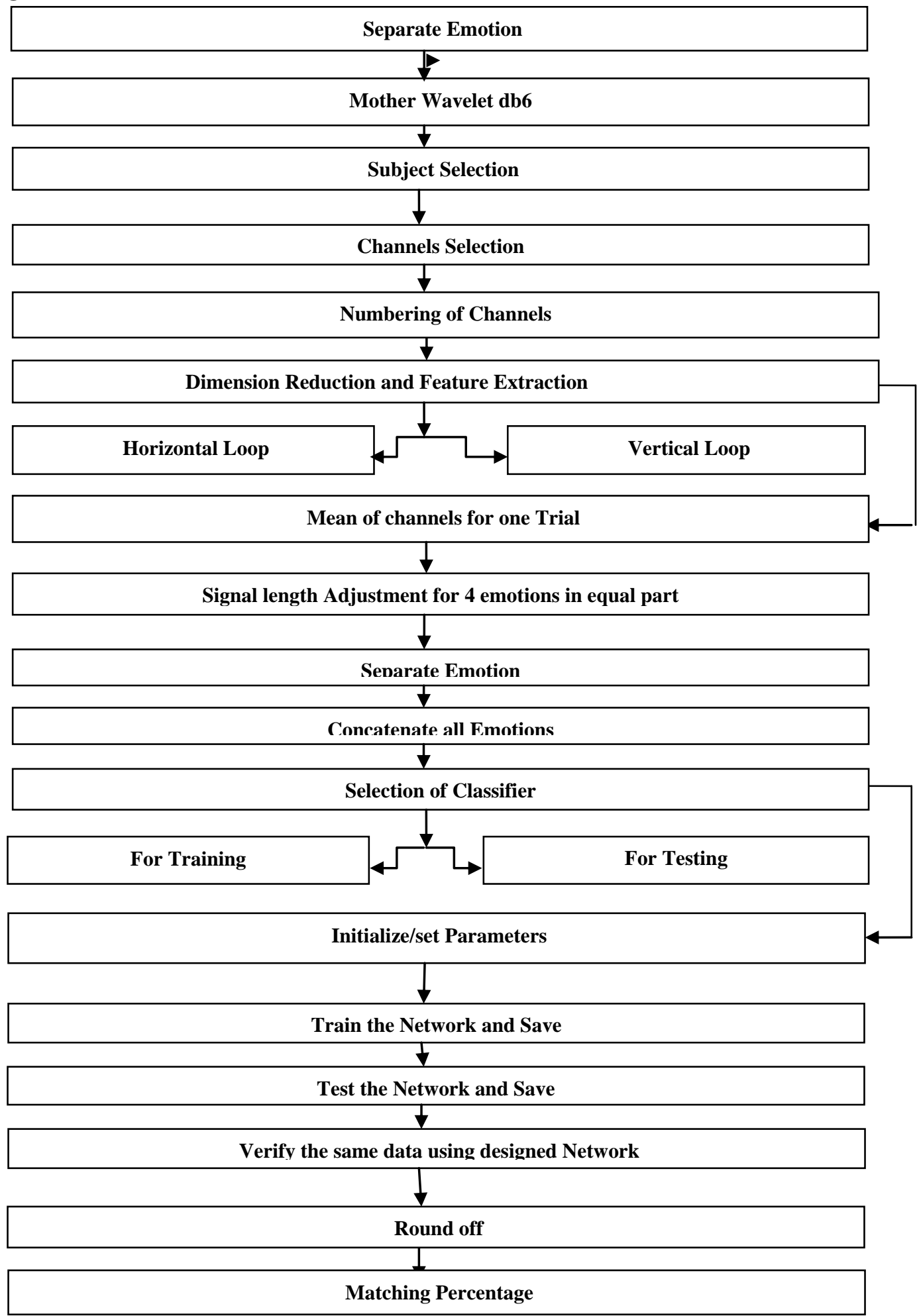

Fig.1Flow Chart of implemented work

\subsection{Description of Flow Chart \\ 1. Separate Emotion}

The data of single object contain more than 4 lakhs. Samples and another problem with data is, it is in form of strip contain for 4 emotions one after another. So separate emotion strip is necessary for single emotion by making matrices of each emotion separation are form like $\mathrm{t} 1, \mathrm{t} 2, \mathrm{t} 3, \mathrm{t} 4$.

2. Feature Extraction

Several families of wavelets are specified in Matlab. We are using here only Daubechies (db6).In db6 two scaling factor are there one is width(A) and another is amplitude (b).By the 
time it compare the signals and providing difference is called coefficient .So the length is now became half the originals. Here the db6 wavelet is used because efficiency of signals is getting much better than any another wavelet. Principle of db6 is easy to understand when the discrete signals are passing from db6 low frequency and high frequency is separate.

\section{Subject Selected}

Load the object, at a time only one object is operated in particular manner.

\section{Select Channel}

Channels selection is necessary because out of 40 channels only 15 selected channels gives the perfect information about brain activity and reaming are useless. These channels are 24 78916182122232425262832 .

\section{Numbering of the Channel}

Taking same index of mention channels, numbering of channels is necessary because it will work according to array numbers.

\section{Dimension Reduction and feature extraction}

Here two loops are operating in single matrix.

For " $r=1$ : size (data, 1$)$; temp=data(r, channels, : )", $r=1$ at the rate of 40 iterations, this happened in $1^{\text {st }}$ loop. selected channels ie. 15, ':' means it will take everything which contain in mention matrix, but it will work for only one plate.

\section{Forsecondloop}

$\mathrm{c}=1: \mathrm{Sc} ; \mathrm{t}=$ reshape(temp $(1, \mathrm{c}$, :), 1 , size(temp,3)), $\mathrm{C}$ is for 15 times ,then reshape the getting matrix for first row ,first column, it is necessary because the matrix is in $1 * 15^{*} 8064$ form ie. Three dimensional. Reshaping is required for total data in single line ie. In t. with concatenate process in s blank matrix which gives single strip 2 dimension figures $15 * 8064$, which contain 4 emotion valence, arousal, dominance and liking. For now it is huge signals so far doing by reduction technique it can be converted into small signal

For reduction discrete wavelet db6 by is used, for first time applying db6 it gives half the number ie, from 8000 signals , 4000 are of approximation coefficient ' $\mathrm{A}$ '(low frequency) and 4000 are of detail ' $B$ ' high frequency ,repeating the same loop high signal get removed again by passing only low frequency , two high and low component were found. These reduction is used for 6 times ie. 6 level decomposition and according to that $15 * 125$ is obtain.

After that the array in vertical concatenation is transform ie.

One after another for 40 trials.

\section{Mean of all channels for one trial}

Taking square mean is best solution and for all channels , because single Wavelets coefficient contain in positive and negative integer that's why arranging in better manner mean is required .Here square means take out energy ie. En

$=\sum \frac{x^{\wedge} 2}{n}$, where $\mathrm{n}$ is 15 , store in en, from the data.

\section{Signal length Adjustment for 4 Emotions in Equal parts}

A strip of four equal parts corresponding to four emotions is needed. It can be form from 124 signals, 31 each part ie. Valance, Arousal, Dominance, Liking is separate. For this another loop considered.

\section{Separate Emotion}

For vertical concatenation first samples ranges from 1-31, second is from 32-62, third from 63-93, fourth from 94-124 in e1, e2, e3, e4arrays accordingly.Adjustment of frames is needed for neural network in new strip .Here horizontal form $31,31,31,31$ ie. each of horizontal concatenation is ie. $t 1$ of valance ,t2 of arousal ,t 3 of dominance, $t 4$ of liking of 40 trials .

\section{Concatenate all emotions}

From 40 trials ie. 32 for train the neural network and 8 for testing the neural network. For training (Ptrain) of 32 signals, we set the system knowing by valance is equal to 1 , arousal is equal to 2,dominance is equal to 3 and liking is equal 4 and expected back same result in Ptest is required for 1-8 signal

\section{Selection of classifier}

Here the data is divided in two parts $75 \%$ of data is used for train the system and remaining $25 \%$ data is used for testing the data. Neural Network used hear as classifier. It's an artificial brain like structure.

\section{Initialize/set parameters}

Consider one neuron which contain weight and bias, neuron output goes to one function and then output achieve, this function is called transfer function. This algorithm is back propagation used in Neural Network when weight, bias changes accordingly the Neural Network is trained. This complete process is performed by LEVENBERG MARQUARDT algorithm using mean square error. This complete process is known as one iteration, providing error up to $10^{\wedge}(-6)$, train the network up to 10 iteration(epoch), Step size or learning rate is 0.1 . Three layers were there for NN accordingly functions like tansigmodial function, log sigmodial function, purelin function transfer function were used, providing specified minimum gradient factor.

\section{Train the network and save}

According to given parameter the network is train and after training the network is save.

\section{Test the test data}

When network is saved the testing signal is given to network and according to specified parameter the output is obtain.

15. Verify the same data using designed Network

Verify or cross check the same output which is given to trained network is corrected or not provided the testing the data is unique.

\section{Round off}

It may happen that some of the number obtain is in the point form so round off is necessary for integer.

17. Compare expected result and NN output previous output has compare with present output. For obtain maximum efficiency and accordingly the neural network output is formed.

\section{Matching Percentage}

Finally the output is in percentage form. This output is vary according to the variation in weight and bias .More the error, then less the efficiency, so if the system train maximum time then output efficiency is much better than previous ,but some time it also happen that system reach to its maximum efficiency and will not give further more efficiency 


\section{DATABASE DESCRIPTION}

DEAP database is used for obtaining the results. These files contain a down sampled (to $128 \mathrm{~Hz}$ ), preprocessed and segmented version of the data present in the Matlab (data preprocessed Matlab.zip) and pickled python/numpy(data preprocessed Matlab.zip) formats. This version of the data is well-matched to those wishing to quickly test a

classification or regression technique in the absence of the hassle of processing all the data first. Each zip file contains 32 .data (python) or .mat (matlab) files, one per participant.

Array name - Data Array shape - 40 x 40 x 8064

Array content - video/trial $\mathrm{x}$ channel $\mathrm{x}$ data

Labels 40x4 video/trial x label (valence, arousal, dominance, liking) The videos are in the order of Experiment_id, so it does not in the order of presentation. This means the first video is the same for each participant. Data was downsampled to $128 \mathrm{~Hz}$. Electrooculogram (EOG) artifacts were removed. A band pass frequency filter from $4.0-45.0 \mathrm{~Hz}$ was applied. This data was averaged to the common reference. The EEG

channels were reordered so that these all are follow the Geneva order given above. The data was segmented into sixty second trials and a 3 second pre-trial baseline removed. The 40 trials were reordered from presentation order to videos.

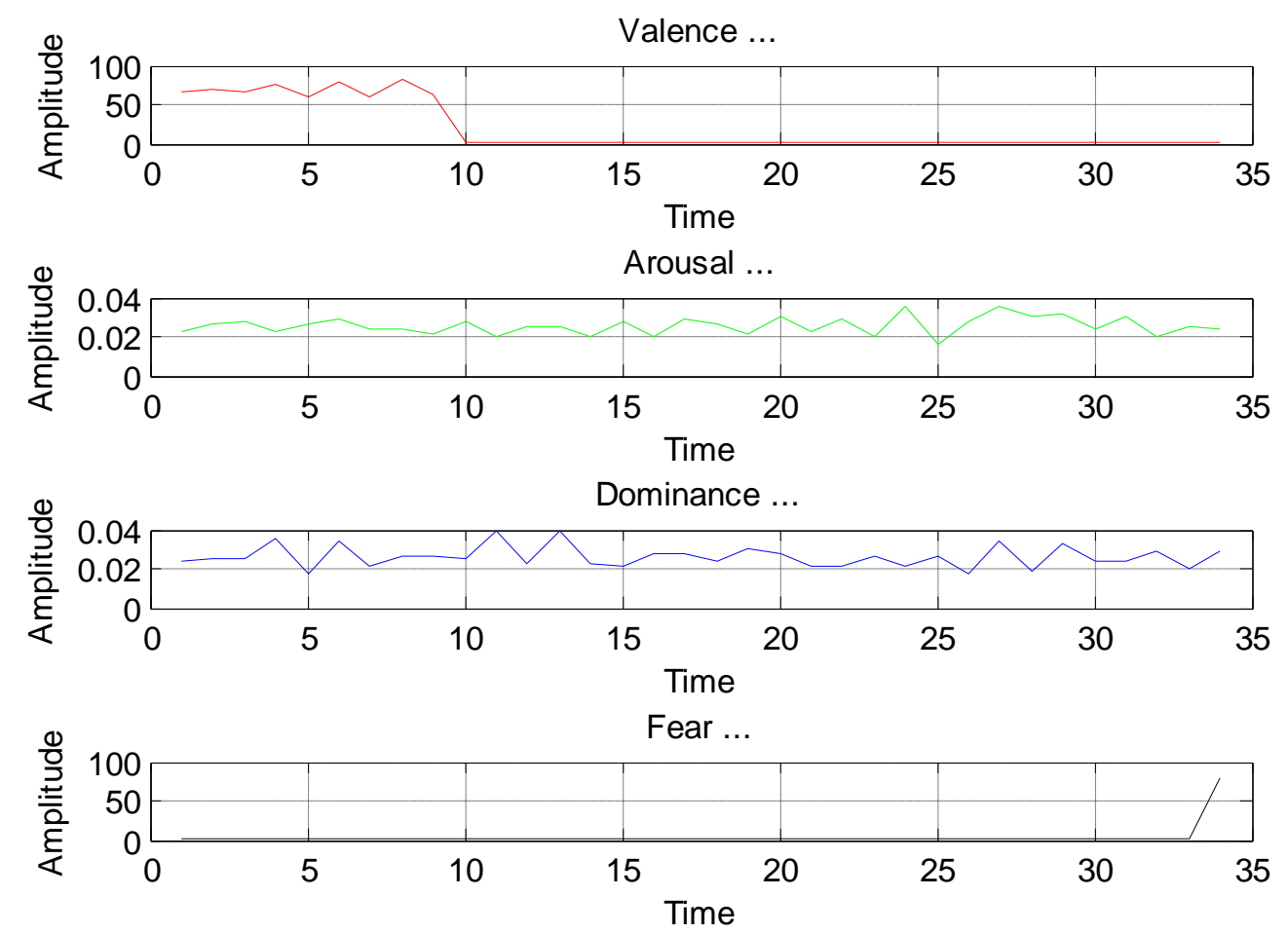

Fig.2 Emotions recording over a EEG channels

\section{RESULTS AND DISSCUSSION}

For acquiring the data the No. of subjects that are used are 32 . 40 channels are used in obtaining the EEG signals. The signals that are obtained are sampled with a sampling frequency of 128 samples/sec. Therefore the total data size is $40 * 40 * 8064$. Out of these 40 channels only 15 channels are selected for obtaining the EEG signals since these 15 channels give the best results. Therefore the data size is reduced. The 8064 samples are divided in four parts where each part corresponds to a particular emotion.

Once all the signals are obtained the data is reshaped. For reducing the data size wavelet reduction method is used. db6 wavelet is used in this project. When db6 is applied once the 8064 samples are reduced to halve. After applying 6 level decomposition number of samples is reduced to 125 . Due to decomposition using wavelet transform, wavelet coefficients are obtained which can be positive or negative and therefore all the coefficients are squared. By taking mean a single frame is obtained for all 15 channels and likewise we get 40 frames for all trials.

All the samples are divided in equal slots for all the four emotions. Vertical concatenation is used to arrange the data in the vertical format. But to feed the data to the neural network it should be the horizontal format and therefore horizontal concatenation is used. Once all the emotions are concatenated it is placed in an separate array. For each emotion $75 \%$ of the samples are used for training the neural and $25 \%$ of it is used for testing. The neural network is trained by setting several parameters. Expected results and neural network results are compared and the outputs are displayed. While downloading, data was downloaded for only 17 subjects and used in this work for obtaining the results. 


\subsection{Four Class Problem}

Table 1. Results of four class problem

\begin{tabular}{|c|c|c|}
\hline $\begin{array}{c}\text { Serial } \\
\text { No }\end{array}$ & Subject & Efficiency (\%) \\
\hline 1 & S01 & 78.125 \\
\hline 2 & S02 & 78.125 \\
\hline 3 & S03 & 75 \\
\hline 4 & S04 & 78.125 \\
\hline 5 & S05 & 75 \\
\hline 6 & S06 & 81.25 \\
\hline 7 & S07 & 75 \\
\hline 8 & S08 & 78.125 \\
\hline 9 & S09 & 84.375 \\
\hline 10 & S10 & 78.125 \\
\hline 11 & S11 & 81.25 \\
\hline 12 & $\mathrm{~S} 12$ & 78.125 \\
\hline 13 & S13 & 78.125 \\
\hline 14 & S14 & 78.125 \\
\hline 15 & S15 & 81.25 \\
\hline 16 & S16 & 75 \\
\hline 17 & S17 & 75 \\
\hline
\end{tabular}

For four class problem, the emotions valence, arousal, dominance, liking we have taken data of 17 subjects after every interval of time and the various output accuracy has been obtained for all the 17 subjects and the highest output accuracy which is obtained is 84.375 . The output accuracy is not $100 \%$ because the emotions arousal and dominance are almost same so the neural network gets confused and the accuracy of $100 \%$ is not achieved here.

\subsection{Three class problem}

Table 2. Results of Three class problem

\begin{tabular}{|c|c|c|}
\hline $\begin{array}{c}\text { Serial } \\
\text { No }\end{array}$ & Subject & Efficiency (\%) \\
\hline 1 & S01 & 70.833 \\
\hline 2 & S02 & 70.833 \\
\hline 3 & S03 & 79.166 \\
\hline 4 & S04 & 78.33 \\
\hline
\end{tabular}




\begin{tabular}{|c|c|c|}
\hline 5 & S05 & 58.33 \\
\hline 6 & S06 & 75 \\
\hline 7 & S07 & 70.833 \\
\hline 8 & S08 & 70.833 \\
\hline 9 & S09 & 66.66 \\
\hline 10 & $\mathrm{~S} 10$ & 66.666 \\
\hline 11 & S11 & 50 \\
\hline 12 & $\mathrm{~S} 12$ & 58.33 \\
\hline 13 & $\mathrm{~S} 13$ & 54.16 \\
\hline 14 & S14 & 66.66 \\
\hline 15 & S15 & 58.33 \\
\hline 16 & S16 & 66.66 \\
\hline 17 & S17 & 62.5 \\
\hline
\end{tabular}

For three class problem, the emotions valence, arousal, dominance we have taken data of 17 subjects after every interval of time and the various output accuracy has been obtained for all the 17 subjects and the highest output efficiency which is obtained is 79.176. The output efficiency is not $100 \%$ because of the same reason as in case of four class problem. Experienced by the subjects during the interaction period with audio-visual content. This study is ongoing to involve various types of classification algorithms in order to track the emotional stage of brain activation during audio-visual environment of stimuli. This study's result gives a framework of methodology that can be used to elucidate the dynamical mechanism for changing of human emotional underlying brain structure.

\section{CONCLUSION}

Here in this paper we proposed an approach for discrete emotion recognition based on the processing of EEG signals. On this research, that there is lack of international standard data base and it is one of the biggest limitations. Therefore, here we are comparing the efficacy of emotion classification with conventional features with the help of newly proposed energy features. Here, the modified energy features is more effective than conventional features to classify the emotions with higher classification rate. Therefore the extracted features are successfully capturing these emotional changes of the subject through their EEG signals regardless of the cultural background of users.
Again, it also helps to shows a significant relationship between EEG signals and emotional states that can be experienced by the subjects during the interaction period with audio-visual content. This study is ongoing to involve various types of classification algorithms in order to track the emotional stage of brain activation during audio-visual environment of stimuli. This study's result gives a framework of methodology that can be used to elucidate the dynamical mechanism for changing of human emotional underlying brain structure.

\section{FUTURE SCOPE}

In future more efficient feature extraction algorithm with the help of various types of wavelet functions and with a various different type of set of statistical features can be developed for improving the emotional classification rate. The EEG signal processing based methodology for emotion classification may be improved further. Research can further be carried on development of a unified algorithm which incorporates various biological signals such as, and Electromyogram (EMG), Electrooculogram (EOG) Electroencephalogram (EEG) so as to implement more natural human computer interface systems. 


\section{REFERENCES}

[1] Bajaj, V.,Pachori, R.B. " Human Emotion Classification from EEG Signals Using Multiwavelet Transform" in Medical Biometrics, International Conference, 2014.

[2] Chai Tong Yuen1,*, Woo San San1, Mohamed Rizon2 and Tan Ching Seong "Classification of Human Emotions from EEG Signals using Statistical Features and Neural Network", International Journal of Integrated Engineering (Issue on Electrical and Electronic Engineering)

[3] Min-ki-Kim1, Miyoungkim 2, Eunmi oh, Sung- Phil, Kim3, "A review on the computational method for emotional state estimation from the human EEG" in review article of international journal of computer science, volume 13, 2013

[4] Murugappan, M., "Human emotion classification using wavelet transform and KNN" in Pattern Analysis and Intelligent Robotics (ICPAIR), International Conference on (Volume: 1), June 2011.

[5] Mallat S. G., "A Theory for multi- Resolution signal decomposition: The wavelet representation", in IEEE transaction on pattern Analysis and Machine Intelligence, vol-11, no.7, 1998, pp-674-693.

[6] Chethan P and Cox M, "Frequency characteristics of wavelet , " in IEEE Transaction on power Delivery, vol17, no.3, 2002, pp 880-886.

[7] Charles W. A. and Zlatko S., " Classification of EEG signals from four subjects during five Mental tasks", in IEEE proceeding on Engineering Application in Neural network, 1997, pp 407-414.

[8] Wanpracha AC, Ya - Ju Fan, and Rajesh CS, “ On the Time series KNN classification of abnormal Brain activity ", in IEEE Transaction -part A: System and Human, vol 37, no 6, ,2007, pp 1005-1016.

[9] P. C. Petrantonokis and L. J. Hadjileontiadis, " Emotions recognition from EEG using higher Order crossing," in Information Technology in Biomedicine, IEEE Transactions on (Volume:14 , Issue: 2 ) Biometrics Compendium, IEEE, October 2009, Pp 186-197

[10] S. K. Hadljidimitriou and L. J. Hadjileontiodis , “ Towards on EEG based recognition of music Linking using Time-frequency analysis", in IEEE Transactions on Biomedical Engineering, (Volume:59 , Issue: 12 ) September 2012, Pp 3498-3570.
[11] J. Kim and E. Andre, " Emotion recognition based on physiological changes in music listening " in IEEE Transactions on Pattern Analysis and Machine Intelligence, IEEE Transactions on (Volume:30 , Issue: 12 ), February 2008, pp 2067-2083.Classification of Human Emotions Using EEG Signals 52

[12] Martinez, A. M.; Kak, A. C "PCA versus LDA". IEEE Transactions on Pattern Analysis and Machine Intelligence 23 (=2): 228-233. doi:10.1109/34.908974

[13] Huy Nguyen and Rong Zheng, "Binary Independent Component Analysis With or Mixtures", IEEE Transactions on Signal Processing, Vol. 59, Issue 7. (July 2011), pp. 3168-3181.

[14] Painsky, Amichai; Rosset, Saharon; Feder, Meir. "Generalized Binary Independent Component Analysis". IEEE International Symposium on Information Theory (ISIT), 2014: 1326-1330.

[15] Wu Jin; Zhang Jiacai; Yao Li, "An automated detection and correction method of EOG artifacts in EEG-based BCI " in Complex Medical Engineering, ICME International Conference, 2009.

[16] M. L. Phillips , W. C. Drevents , S. L. Rouch and R. Lane, " Neurobiology of emotion perception II : Implementation for major psychiatric disorders", in Biological psychiatry ,vol: 54, no.5, 2003, pp 515-528.

[17] L. F. Barrett , " Discrete emotions or dimensions the role of valence focus and arousal focus ." in Cognition and Emotions, vol 12, no. 4 , 1998, pp. 579-599.

[18] R. Adolph's , "Neural system for recognizing emotion," in current opinion in Neurobiology , Vol-12, no. 2, 2002, pp.169-177. repetition , and time- no -task, " Biological Psychological vol 75 no.1 pp. 101-108, 2007.

[19] J. K. Olofsson and J. Polich, "Affective visual event related potential, arousal.

[20] Xue J. Z. , Zhang H, Zheng c-x, Yan X-G, "wavelet packet transform for feature extraction of EEG during mental tasks", in International conference on machine learning and cybernetics Vol-3, pp-360-363.

[21] Murugappan M, Rizon M, Nagrajan R, Yaccab s, “ An investigation on visual and audio-visual Stimulus based emotions recoginition using EEG", in International Journalof soft computing and Application (IJSCA) EURO Jauranal , vol.48, no.2, $\mathrm{pp}=281-299$. 